\title{
3 Research Square \\ Genome-wide identification, structural analysis and expression profiles of SRS gene family in quinoa
}

\author{
xiaolin zhu \\ Gansu Agricultural University \\ baoqiang wang \\ Gansu Agricultural University \\ xian wang \\ Gansu Agricultural University \\ xiaohong wei ( $\square$ 1981263554@qq.com ) \\ Gansu Agricultural University https://orcid.org/0000-0001-7869-6042
}

\section{Research Article}

Keywords: Quinoa, SRS gene family, Evolutionary analysis, Expression Pattern

Posted Date: September 3rd, 2021

DOI: https://doi.org/10.21203/rs.3.rs-839802/v1

License: (a) (1) This work is licensed under a Creative Commons Attribution 4.0 International License. Read

Full License 


\section{Abstract}

Based on the whole genome data information of quinoa, the CqSRS gene family members were systematically identified and analyzed by bioinformatics methods, and the responses of CqSRS genes to $\mathrm{NaCl}(200 \mathrm{mmol} / \mathrm{L}), \mathrm{SA}(200 \mathrm{umol} / \mathrm{L})$ and low temperature $\left(4^{\circ} \mathrm{C}\right)$ were detected by qRT-PCR. The results showed that a total of 10 SRS genes were identified in quinoa, and they were distributed on 9 chromosomes, and there were 4 pairs of duplicated genes. The number of amino acids encoded ranged from 143 to 370 , and the isoelectric point ranged from 4.81 to 8.90 . The secondary structure was mainly composed of random coil(Cc). Most of the CqSRS genes were located in the cytoplasm (5 CqSRS). Phylogenetic analysis showed that the CqSRS gene was divided into three evolutionary groups, and the gene structure showed that the number of exons of CqSRS was between 2-5. Promoter analysis revealed that there are a total of 44 elements related to plant hormone response elements, light response elements, stress response elements and tissue-specific expression in the upstream of the gene. Protein interaction showed that all $10 \mathrm{CqSRS}$ proteins appeared in the known protein interaction network diagram in Arabidopsis. Expression profile analysis showed that CqSRS genes had different expression patterns, and some genes had tissue-specific expression. qRT-PCR showed that all SRS family genes responded to SA, $\mathrm{NaCl}$ and low-temperature treatments, but the expression levels of different CqSRS genes were significantly different under various stresses. This study lays a foundation for further analyzed the function of CqSRS family genes.

\section{Introduction}

In the process of plant growth and development, plants will encounter a variety of abiotic stresses (drought, salt, low temperature, high temperature), as well as biological stresses such as bacteria and fungi, which have a great impact on plant yield and quality. Thus, to adapt to extreme environments, plants change at the genome-wide level to resist abiotic stresses. Transcription factors (TFs), also known as cis-acting elements, are critical to this process and can specifically bind to the promoter of target genes. The structure and function of TFs play a key role in regulating plant resistance to biotic and abiotic stresses (Boyer et al. 1982). SHI related sequence (SRS) gene family, also known as short internodes (SHI) or SHI/STY/family of SRS (for short internodes, stylish, and SHI-related sequence), is unique in plants. The family encodes a specific transcription factor with two different conservative areas, and the prediction proteins show the sequence of particularly high consistency in two areas, the first area is located in the protein amino acid sequence of $\mathrm{N}$-terminal. The results show that the supposed ring domain contains 31 amino acid residues in the consistent sequence of Cys-X2-Cys-X7-Cys-X-His-X2-Cys-X2-Cys-X7-Cys-X2-X2-His. The motif is a $\mathrm{C} 3 \mathrm{HC} 3 \mathrm{H}$ ring domain and the domain is conservative ring zinc finger. The second conserved domain is located at the $\mathrm{C}$-terminal, which is the only domain of proteins in the $\mathrm{SHI}$ family. This domain has four highly conserved residues, so it is named IGGH. In addition to these two conserved domains, the remaining protein sequences are highly differentiated (Fridborg et al. 2001), and these characteristics are particularly critical for their transcription factor functions. The SRS genes of Arabidopsis thaliana contain two conserved domains, zinc finger domain and IGGH domain, but the sequence is also highly differentiated. SHI is the first member of the SRS gene family and has been identified in Arabidopsis dwarf mutant SHI. This gene can inhibit GA response at the GA biosynthetic site, and the SRS/STY protein contains acidic 
amino acids, which is a characteristic of this family of proteins as transcriptional activators (Fridborg et al. 1999).

At present, a total of 11 SRS genes have been identified in maize (He et al. 2020), and 11 SRS genes in Arabidopsis, including SHI, STY1, STY2, LRP1 and SRS3-SRS8 (Kuusk et al. 2006; Sohlberg et al.2006). Many SRS genes play important roles in regulating plant hormone biosynthesis, photomorphogenesis, metabolization-related material structure, signal transduction, and plant organ growth and development. For example, during the development of lateral roots (LR), LRP1 is regulated by the auxin signal transduction mechanism (Ive et al. 2008; Bert et al.2012), and auxin and histone deacetylation affect the expression of $\angle R P 1$, and by regulating the dynamic balance of auxin in Arabidopsis thaliana. Meanwhile, it negatively regulates the development of $L R P$ in the downstream of the auxin reaction module of $L R$, and it plays a role in the downstream of rootless and undetectable meristematic tissue 1 ( $R U M 1), R U M 1$ is an Aux/IAA protein that regulates the crown root development of corn (Zhang et al. 2015). STY1, STY2 and STY3 in LotuS Japonicus, as direct LiNF-YA1 targets, are involved in the formation of nodules (Hossain et al. 2016). STY1 up-regulates auxin biosynthesis (Eklund et al. 2010). Recent studies have shown that Arabidopsis SRS5 gene is a positive regulator of photomorphogenesis, which can directly bind to promoters of photomorphogenesis genes (such as $H Y 5, B B X 21$ and $B B X 22$ ) to activate its expression to promote photomorphogenesis. Meanwhile, SRS5 is also a target of COP1-mediated degradation (Yuan et al. 2018). The SHI/ STY/ SRS genes play a conservative role in the apex of Arabidopsis regulatory network, and these genes guide the development of styles and stigmas (Gomariz et al. 2017). Studies in rice showed that OsSHI1 inhibited the transcriptional activity of IPA1 and regulated plant structure by affecting the DNA binding activity of IPA1 on the promoter region of OsTB1 and OsDEP1 (Duan et al. 2019).

So far, there have been many studies on the identification and functional analysis of SRS gene family in Arabidopsis (Greb et al. 2003), followed by studies on maize (He et al. 2020). By contrast, the SRS gene has yet to be reported in quinoa (Chenopodium quinoa Willd.), which has more nutritional value than any traditional food crop. Besides, quinoa is suitable for growing in high altitude areas $(>3,500 \mathrm{~m}$ above sea level), and it is resistant to multiple abiotic stresses, including cold-tolerant, drought-tolerant, salt-tolerant and barren-tolerant. It has the potential to provide a highly nutritious food source that can be grown on marginal lands not currently suitable for other major crops (rice and maize). It is regarded as a facultative halophyte and shows a strong resistance to drought and low temperature as well. The value is as proteinrich as beef, and quality is as good as meat and milk proteins. However, despite its agronomic potential, quinoa is still an underutilized crop, with relatively few active breeding programs. Breeding efforts to improve the crop for important agronomic traits are needed to expand quinoa production worldwide. Currently, the lack of breeding for specific environments, the high photoperiodic sensitivity and the relatively low yield are the major factors that limit quinoa cultivation in nonnative areas. SRS transcription factors control a diverse range of developmental processes in plant, including root formation, leaf development, floral induction and flower development, and photomorphogenesis. and the recent publication of quinoa genome provides an opportunity to identify the SRS genes of quinoa (Jarvis et al. 2017). Therefore, in this study, we identified ten SRS genes in quinoa, and systematically analyzed it from the basic physical and chemical properties, phylogeny, gene duplication, tissue expression, protein interaction and other aspects of 
the members of the gene family. These results provide a reference for further study on the function of SRS genes in quinoa, and provide a certain theoretical basis in breeding of quinoa.

\section{Materials And Methods}

\subsection{Search and identification of SRS gene members of quinoa}

The quinoa genome database (Chenopodium quinoa v1.0), including coding sequences, protein sequences and other information were downloaded from Phytozome v12

(https://phytozome.jgi.doe.gov/pz/portal.html). The amino acid sequences of the known Arabidopsis SRS family members were downloaded from the Arabidopsis Information Resource (TAIR)

(http://www.arabidopsis.org) (Poole et al. 2007) database, and SRS genes in quinoa were obtained by using their amino acid sequences for homologous alignment and removing redundant sequences. This screening was then combined with the SRS domain. Prediction of protein conserved domains using PFAM (http://pfam.xfam.org/family) (Finn et al. 2014), NCBI-CDD (https://www.ncbi.nlm.nih.gov/cdd/) (Marchler et al. 2011) and SMART (http://smart.embl-heidelberg.de) (Schultz et al. 2000).

1.2 Basic physical and chemical properties of proteins and phylogenetic analysis

The basic physical and chemical properties of SRS proteins in quinoa were analyzed by ExPASy (https://web.expasy.org/protparam/) (Gasteiger et al. 1999), and the subcellular localization was predicted by Psort-Prediction囚http://psort1.hgc.jp/form.html(Gardy et al. 2005).

Phylogenetic trees of SRS family proteins of Arabidopsis, maize, tomato, spinach and quinoa were constructed by using Clustal X version 2.1 (Larkin et al. 2007) in MEGA7 (Kumar et al. 2016). ZmSRS SoSRSロSISRS genes come from PlantTFDB v5.0 (Tian et al. 2019). We use Poisson mode, data missing was set as pairwise deletion, repetition number is 1000 , other parameters are default. Evolutionary tree beautification through Evolview囚https://evolgenius.info//evolview-v2/\#login囚(Zhang et al. 2012).

\subsection{Gene structure and conserved motifs analysis}

Based on the Gff annotation of the quinoa genome, the gene structure of the exon/intron of SRS genes were constructed by using the Gene Structure Display Server (GSDS) (http://gsds.cbi.pku.edu.cn/) (Huang et al. 2015). Multiple Em for Motif Elicitation (MEME) program (http://meme-suite.org/tools/meme) (Steven et al. 1996) was used to analyze the conserved motifs in the amino acid sequence of CqSRS proteins. The number of motif searches was set as 10 , and other parameters were default.

1.4 Chromosomal location and gene duplication analysis

The annotation information of the SRS genes in the quinoa database was used to determine the chromosomal location of members of the family. Fragment duplication pairs are detected on the plant genome duplication database server (http://chibba.agtec.uga.edu/duplication/). The amino acid sequence of partially duplicated CqSRS genes was predicted by Clustalw software. DnaSP v5.0 software (Librado et al. 2009) is used to estimate of synonymous (Ks) and non-synonymous (Ka) replacement rate (Suyama et 
al. 2006), using the following formula to determine CqSRS gene duplication of time (millions of years ago, MYA) and divergence of time: $T=K s / 2 \lambda \llbracket \lambda=6.56 \mathrm{E}-9 \otimes($ Lynch et al. 2003).

\subsection{Cis-acting element analysis and construction of protein interaction network}

According to the quinoa genome database, $2000 \mathrm{bp}$ upstream of the transcriptional initiation site of SRS family gene was extracted by sequence extraction with TBtools (Chen et al. 2020), which was used as the promoter region of regulation, the cis-regulatory element of the promoter region of the SRS gene was retrieved and analyzed using the PlantCARE (http://bioinformatics.psb.ugent.be/webtools/plantcare/html/) (Lescot et al. 2002). Based on Arabidopsis thaliana. The protein-protein interaction network of quinoa was further predicted by STRING software (https://string-db.org/) (Szklarczyk et al. 2015) (confidence greater than 0.8).

1.6 Secondary structure analysis and tertiary model prediction

The secondary structure of SRS proteins were analyzed on the prediction of secondary structure by NPS@: GOR4 (https://npsa-prabi.ibcp.fr/cgi-bin/npsa_automat.pl?page=/NPSA/npsa_gor4.html) (Combet et al. 2005). At the same time, we predict the tertiary structure of the protein by the Swiss-model server (Kelley et al. 2015).

\subsection{Plant materials and treatments}

Quinoa cultivar safflower dajing yuan (HHDJY) was used as the material. The quinoa was disinfected in $10 \%$ sodium hypochlorite for $20 \mathrm{~min}$, then rinsed with sterile water for 5 times, and seeded on MS solid medium. It was cultured in a greenhouse at $24 \pm 1^{\circ} \mathrm{C}$ for $14 / 10$ hours in light/dark light cycle until germination. The germinated seeds were planted in a 1:1:1 tray containing sand, perlite and peat, and cultured in the growth chamber (relative humidity $60-70 \%$, illumination time $12 \mathrm{~h}$, day-night temperature $28^{\circ} \mathrm{C} / 18^{\circ} \mathrm{C}$ ). After the seedlings had grown for about 2 months, they were placed in an incubator at $4^{\circ} \mathrm{C}$ for low-temperature treatment. Under salt stress, $100 \mathrm{mmol} / \mathrm{L} \mathrm{NaCl}$ was sprayed on the surface of plant leaves. In ABA treatment, 200uM ABA was sprayed on the surface of plant leaves. CK was the plant under normal growth conditions. The stem was collected at $0 \bigotimes 2 \bigotimes 4 \rrbracket 8$, and 12 hours after treatment. And three biological replicates were conducted at each time point. The collected leaves were temporarily stored in liquid nitrogen, and then stored in a refrigerator at $-80^{\circ} \mathrm{C}$ for the subsequent quantitative test.

1.8 Expression analysis of SRS gene, RNA extraction and real-time quantitative PCR (qRT-PCR)

The SRS gene expression data of quinoa were obtained from transcriptomic data of different tissues and organs of quinoa (No.: PRJNA394651) and the aboveground tissues of quinoa seedlings under drought, high temperature, salt and low phosphorus stress (No.: PRJNA306026). RNA-sequencing (RNA-seq) data (PRJNA394651 and PRJNA306026) were downloaded from the National Center for Biotechnology Information (NCBI, https://www.ncbi.nlm.nih.gov/) (Zou et al. 2017). We standardized the data using the Log2 method. 
Total RNA was extracted from each sample using the Trizol total RNA extraction kit (Sangon, Shanghai, China, SK1321), and cDNA was prepared using the Superscript ${ }^{T M}$ III reverse transcriptase kit (Invitrogen). qRTPCR was designed using Primer Premier 5 (Livak et al. 2001). And normalized against Elongation Factor 1 alpha (EF1a) gene transcript levels. The concentration and purity of RNA and CDNA extracted were determined by a quantitative ultraviolet Spectrophotometer Q5000 (UV-VIS), and q-RT-PCR analysis was done with $2 \times$ lyect-SYbr-green-Pcr-mix (Qiagen) in the real-time PCR system of American Applied Biosystems, the program is shown as follows: Denaturation at $95^{\circ} \mathrm{C}$ for $3 \mathrm{~min}$, followed by denaturation at $95^{\circ} \mathrm{C}$ for $10 \mathrm{~s}$ for 40 cycles, and finally annealing/extension at $60^{\circ} \mathrm{C}$ for $1 \mathrm{~min}$ (Zhang et al. 2013). The actin gene was used as the endogenous control. Relative gene expression level was calculated using the $2^{-\Delta \Delta C t}$ method (Livak et al. 2001). Each experiment was repeated in triplicate using independent RNA samples.,

\subsection{Statistical analysis}

Data quantified from the qRT-PCR of the three biological replicates were analyzed with two-way ANOVA using SPSS (version 19) and statistically evaluated using the Duncan method. A difference was considered statistically significant at $\mathrm{P}<0.05$.

\section{Result}

\subsection{Basic physical and chemical properties}

Finally, we identified 10 SRS genes in quinoa, which were named SRS01-SRS10. The coding sequence (CDSs) of the members of this family is between $432-1113$ nucleotides, and the coding amino acid sequence is between 143-370 amino acids (Table 1), with an average of 244 amino acids. Except for CqSRS01 and CqSRS02, the PI of the coding proteins is less than 7, and the hydrophobicity index of all CqSRS proteins is less than 0 , indicating that these proteins are hydrophilic. Subcellular localization prediction showed that most CqSRS genes were located in the cytoplasm (5 CqSRS genes), and a few were located in the nucleus, plasma membrane and mitochondria. The structure and stability of CqSRS proteins are determined by the instability index, which provides an estimate of protein stability. In this study, 6 CqSRS proteins were unstable, with the instability index greater than 40. 4 CqSRS proteins may be stable, with an index between 32.45 and 39.45.11.

\subsection{Evolutionary Relationships and Classification of SRS Genes}

To study phylogenetic relationships between the SRS proteins of quinoa, we constructed phylogenetic trees from 44 protein sequences of Arabidopsis (11), maize (9), tomato (9), spinach (5) and Quinoa (10). According to the topological structure of the tree, all plants share a common ancestor with the SRS genes. Meanwhile, according to their homology, they are divided into 3 subfamilies (Fig 1 and S1 Table). The first group has 11 SRS genes, the second group contains 10 genes, and the third group is the largest, containing 23 genes. At the same time, we can observe that there are 14 pairs of orthologous genes in these 5 species (6 pairs in quinoa, CqSRS06/SISRS07, CqSRS04/SISRS03, CqSRS05/AtSRS06, CqSRS02/AtSRS01, CqSRS08/ZmSRS05), and 5 pairs of paracentric homologous gene pairs (2 pairs in quinoa: CqSRS03/CqSRS09, CqSRS01/CqSRS10). There are 2 pairs of homologous genes between quinoa and 
Arabidopsis, indicating that there is no obvious difference between these two species in the evolutionary process.

\subsection{Chromosomal location and gene duplication analysis}

To verify the relationship between genetic differentiation and gene replication, we identified the chromosomal locations of CqSRS genes (Fig 2). In this study, the chromosomal locations of CqSRS gene family members were obtained through the quinoa genome (David et al. 2017). Ultimately, 10 CqSRS genes were located on the 9 chromosomes of quinoa (quinoa has a total of 18 chromosomes), with 1 SRS gene on 8 chromosomes except chromosome 9 , which contains 2 SRS genes.

The replication of CqSRS genes was further tested. Previous studies showed that 5 or fewer genes located within the range of $100 \mathrm{~kb}$ on the same chromosome are usually considered as tandem repeats (McGregor et al. 2017), so there are no tandem repeats in this study. We based on two conditions (comparison rate of two genes $>75 \%$, comparing similarity $>75 \%$ ), screening for repeat genes, identified to 4 of duplicated genes (Table 2), and they respectively located on different chromosomes and thus belongs to the duplicated gene fragments, and repeated occur between 6.830-14.151 MYA. The history of the selection acting on the coding sequence can be measured in terms of the ratio of non-synonymous substitutions to synonymous substitutions $(\mathrm{Ka} / \mathrm{Ks}) . \mathrm{Ka} / \mathrm{Ks}<1$ was selected for purification. When the two sequences drift in neutral and special, $\mathrm{Ka} / \mathrm{Ks}=1$. At specific sites of positive selection, $\mathrm{Ka} / \mathrm{Ks}>1 . \mathrm{Ka} / \mathrm{Ks}$ values of 4 gene pairs in this study were all less than 1 , indicating that the evolution of all gene pairs was mainly influenced by purification selection, and purification selection could inhibit the differentiation of duplicate genes.

\subsection{Analysis of gene structure and conserved motifs}

On the one hand, the diversity of gene structure reflects the evolutionary relationship of gene families. Meanwhile, the intron-exon pattern plays a key role in gene function. Therefore, we analyzed the exon/intron pattern of members of this family by comparing the coding sequence with the corresponding genomic DNA sequence. Results showed that the number of exon CqSRS between 2 and 5 , and at the same subfamily gene has a similar introns/exon mode. For example, the number and length of exons of corresponding genes in subfamilies 1, 2 and 3 are highly similar, and the genes are highly homologous to each other, suggests that they are in the process of evolution is derived from a common ancestor, or maybe the result of a genetic replication (Fig 3). The conservative motifs of CqSRS proteins were analyzed by using MEME and 10 conserved motifs were selected. It was found that Motif 4 exists in all CqSRS genes, Motif 1 exists in most CqSRS genes, and Motif 3, 5, 6 and 8 only exist in CqSRS03 and CqSRS09. Motif 9 may be the basis for the division of CqSRS01 and CqSRS02 in the same branch. Most CqSRS genes with similar gene structure have the same motif compositions and similar functions.

\subsection{Cis-acting element analysis and construction of protein interaction network}

In order to study the cis-acting elements in the CqSRS genes promoter region, we analyzed the promoter sequence of CqSRS genes (2000bp upstream of translation starting point) by PlantCARE. We found that all CqSRS genes promoter region contained one or more TATA-box. At the same time, we found a total of 44 
elements related to plant hormone response elements, light response elements, stress response elements and tissue-specific expression in the upstream region of the promoter (Fig 4 and S3 Table). The light response element was the most cis-acting element, followed by plant hormone and stress response element, and the tissue-specific expression element was the least. Plant hormones such as auxin, abscisic acid, gibberellin and jasmonic acid play a key role in plant resistance to adversity. In this study, CqSRS genes contained a variety of hormone-related elements. ABRE, CGTCA-motif, TGACG-motif and other plant hormone elements existed in all CqSRS genes in the form of a single copy or multiple copies. Some genes (CqSRS02, CqSRS05, CqSRS07, CqSRS06 CqSRS07, CqSRS08 and CqSRS10) contained 5 hormone response elements, including abscisic acid (ABRE), AuxRE (AuxRE, AUXRR-core, CGTCA-motif and TGA-Box), salicylic acid (TCA-element), gibberellin (GARE, P-box and TATC-Box) and methyl jasmonate (TGACG-motif). CqSRS genes also contain some tissue-specific elements, including meristem expression elements (CATbox) and endosperm expression elements (GCN4_motif and AACA-motif). In addition, the family also contain a small number of stress response elements, including low-temperature response elements (LTR), drought induction elements (MBS), and defense and stress response elements (TC-rich repeats).

To further investigate which protein interact with SRS family members, a network of protein interaction between quinoa and Arabidopsis was predicted using the STRING database. As can be seen from the figure below, 10 CqSRS proteins appear in the known Arabidopsis protein interaction network (Fig 5). Among them, the protein sequence of AtSTY1 is highly similar to that of CqSRS07, AtSTY1 gene, as a transcriptional activator, can bind to the DNA on $5^{\prime}$-ACTCTAC $3^{\prime}$ and promote the expression of auxin homeostasis regulation genes (such as YUC gene), as well as genes affecting stamen development, cell amplification and flowering time, so CqSRS07 gene may have a similar function. AtLRP1 gene has been identified as an auxininduced gene, and its expression is regulated by histone deacetylation, so the expression of CqSRS01 and CqSRS02 may also be regulated by auxin signal (Singh et al. 2020). 5 CqSRS genes (CqSRS04, CqSRS05, CqSRS06, CqSRS08 and CqSRS10) are similar to AtSHI gene, revealing their synergistic effect with other related proteins (NGA3 and YUC1) to regulate pistillate, stamen and leaf development in a dose-dependent manner and control apical basal configuration, and promote pistil development and stigma formation, and affect the development of blood vessels during pistil development.

\subsection{Secondary structure analysis and tertiary model prediction}

In order to better understand the structural characteristics of CqSRS proteins, a third-level model of the protein family was predicted using Swiss-model, and the results showed that members in the same

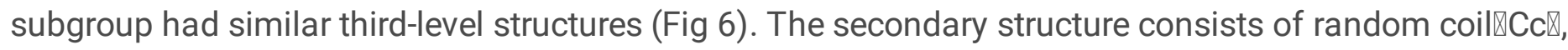

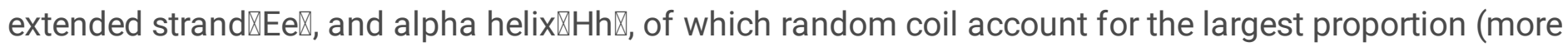
than $50 \%)$ (S4 Table).

\subsection{RNA-seq analysis}

We used transcriptome data to study the expression patterns of genes in this family. The results of heatmap showed that most CqSRS genes showed a low expression under different treatments (Fig 7 and S5 Table). For example, CqSRS04, CqSRS05, CqSRS06 and CqSRS10. CqSRS01, CqSRS02 and CqSRS03 genes are highly expressed in roots under high temperature, low phosphorus, drought and salt stress, and these genes 
may play a key role under abiotic stress. In addition, the expression of CqSRS genes in tissues and organs at different development stages of quinoa was also significantly different. Almost all the genes high expression in Apical meristems and Flowers of white sweet quinoa. Most genes (except CqSRS08) are low expression in leaves. The expression pattern of CqSRS08 was different from that of other proteins. The expression of CqSRS08 was high in all tissues, especially in leaves up to 43, indicating that some SRS genes have the characteristics of tissue expression.

\subsection{Expression profiling of CqSRS genes in different treatments}

Stress seriously affects the growth and development of plants, so qRT-PCR was used to analyse the expression patterns of the family members in roots under stress (Fig 8 and S6-S7 Tables). The results showed that all SRS family genes were responsive to $\mathrm{SA}, \mathrm{NaCl}$ and low-temperature treatments. The expression levels of different CqSRS genes were significantly different under different stress. In SA treatment, some genes (CqSRS02, CqSRS03, CqSRS05 and CqSRS06) showed the same pattern of first increasing and then decreasing, and some genes (CqSRS01, CqSRS04, CqSRS07, CqSRS08, CqSRS09 and CqSRS010) showed the lowest expression after $8 \mathrm{~h}$ treatment. Under $\mathrm{NaCl}$ and low-temperature treatment, most of the genes had the same expression pattern ( $2 \mathrm{~h}$ or $12 \mathrm{~h}$ expression level was extremely significant), and the expression level of the treatment was significantly higher than that of the control group. However, the expression of CqSRS10 gene in $\mathrm{NaCl}$ and low temperature was lower than that in control. These results showed that the CqSRS gene family members in most roots were strongly induced by $200 \mathrm{mmol} / \mathrm{L} \mathrm{NaCl}$, $200 \mathrm{mml} / \mathrm{L} \mathrm{SA}$ and $4^{\circ} \mathrm{C}$ treatment under different treatments, and only a few members were not sensitive to abiotic treatment.

\section{Discussion}

Plants encounter various biological and abiotic stresses during their growth and development, and transcription factors play an important role in a series of biological processes throughout the life cycle of plants (especially in adversity). As an important transcription factor, SRS is significantly characterized by a conservative ring-finger zinc finger domain at the N-terminal of the protein, many SRS genes take part in apical gynoecium development and mediate stigma development (Gomariz et al. 2017). Meanwhile, research showed SRS genes (SHI) are correlated with crop yields (Kuusk et al. 2006). However, the identification of the SRS gene family has only been reported in maize, rice and Arabidopsis thaliana (Kuusk et al. 2006; Yang et al. 2020). It has not been reported in quinoa. Quinoa is a highly resistant crop, and its genome sequencing can help us to identify the resistance genes and improve the genetic improvement of the crops. Therefore, in this study, we identified 10 SRS genes from quinoa, which was consistent with the results of Arabidopsis thaliana (11 SRS) (He et al. 2020) and maize (11 SRS) (Kuusk et al. 2006), indicating that the number of the SRS gene family in different species was not significantly different, and also reflected that there was no large-scale genome-wide replication of these three species after separation. Moreover, the number of amino acids, isoelectric point and molecular weight of the family members' proteins are significantly different, which may be due to the different functions of the family members during their growth and development. To investigate the phylogenetic relationships among members of the SRS family, we construct phylogenetic trees used SRS proteins from Arabidopsis, spinach, tomato, maize, 
and quinoa. Through phylogenetic classification, 44 SRS genes of five species can be divided into three categories, and three groups all contains five species of SRS genes, and illustrate the five species may be derived from a common ancestor. The distribution of SRS genes in maize, Arabidopsis, tomato, spinach and quinoa may be the result of gene differential amplification after the differentiation of monocotyledons and dicotyledons from the same ancestor. At the same time, the number of SRS genes in the 5 species was relatively small, indicating that the retention and duplication of genes in different species were basically consistent with similar evolutionary constraints (Airoldi et al. 2012). 10 SRS genes were distributed on 9 chromosomes, and no tandem duplication was found, which was consistent with the study on SRS family genes in maize (Kuusk et al. 2006).

In order to understand the structural diversity of SRS genes in quinoa, the intron/exon structure was analyzed. Previous research has shown that an intron-rich gene can lose multiple introns simultaneously, resulting in an intron-free genetic ancestor, and the intron-free genes in eukaryotic genomes may be derived from the horizontal gene transfer of ancient prokaryotes (Wang et al. 2019). The different splicing states of exons and introns may be meaningful to the evolution of CqSRS genes. In this study, the number of introns in subgroups 1, 2 and 3 were small and similar, which may be due to intron loss during the evolution of the SRS genes, subgroups 4 and 5 have similar intron numbers. Although introns have no effect on protein sequence, their relative positions provide clues to predict how genes and their corresponding proteins evolve and further promote the structural diversity of genes (Rogozin et al. 2000). This diversity of gene structure may drive the evolution of gene families. And may enable genes to have new functions that can help plants better adapt to environmental changes (Fan et al. 2014). Meanwhile, we identify 10 conserved motifs and the CqSRS genes in the same subfamily were found to have the same motif composition, which indicates that the genes of the same subfamily have similar functions. Although the $10 \mathrm{SRS}$ genes share a common conserved motif 4 , they also have their unique conserved motifs, and different motif composition may contribute to the functional diversity of CqSRS members (Liu et al. 2015). The study on the SRS gene structure and conserved motif of quinoa provides a reference for further study on the evolution of the SRS family of quinoa.

Recent studies have shown that gene replication not only is important in the expansion and rearrangement of genomes in the evolutionary process, but also induces the diversification of gene functions (Zhang et al. 2013). The three most important evolutionary patterns are fragment repetition, tandem repetition, and transposition events (Mao et al. 2016). 5 or fewer genes located within the $100 \mathrm{~kb}$ range of a chromosome are generally considered tandem repeats, while gene duplication occurring on different chromosomes is considered fragment duplication (Liu et al. 2011; Hu et al. 2015). In order to elucidate the amplification mechanism of the SRS gene family in quinoa, we studied the phenomenon of the SRS gene family replication, and the results showed that 10 CqSRS genes were distributed on 9 chromosomes. In addition, there are 4 pairs of gene duplication, but chromosomal positioning shows that these genes are located on different chromosomes, so these 4 pairs of genes belong to fragment duplication rather than tandem duplication in the process of evolution. In addition, $\mathrm{Ka} / \mathrm{Ks}$ (non-synonymous and synonymous substitution ratio) of these 4 pairs of genes were all less than 1, indicating that purification selection plays a major role in the expansion of SRS genes in quinoa, which is consistent with previous studies (Cao et al. 2019). Meanwhile, these duplicated genes may have retained ancestral functions during evolution. 
Cis-acting elements are important in plant defense against various biotic and abiotic stresses (Zhao et al. 2016), and they can specifically bind with transcription factors to regulate gene transcription (Riechmann et al. 2000). In this study, we identified several cis-acting elements associated with auxin, gibberellin, salicylic acid (SA), abscisic acid (ABA), and methyl jasmonate (MeJA) in the promoter region of the CqSRS genes. These hormone response elements play a key role in various life activities in plants. ABRE (ABA response elements, 40), CGTCA-motif (MeJA response elements, 22), and TGACg-motif (MeJA response elements, 22) were found in all CqSRS genes, indicating that these elements are highly conserved in the CqSRS family. At the same time, virtually all SRS genes contain two or more identical copies of the cis-acting elements. This may play a role in enhancing regulation of gene transcription and adapting to environmental changes. ARE is necessary for anaerobic induction and exists in multiple copies of all CqSRS genes. The analysis of CqSRS genes promoter region revealed the existence of various cis-acting elements, which regulated the expression level of genes.

The analysis of CqSRS tertiary structure and protein-protein interaction is helpful to further understand the function of CqSRS genes. In this study, genes in the same branch have similar protein structures, such as CqSRS07 and CqSRS10, so they may have similar functions. Furthermore, we constructed a network of protein interaction between Arabidopsis and quinoa. Previous studies have shown that AtLRP1 gene has been identified as an auxin induced gene, and its expression is regulated by histone deacetylation, so the expression of CqSRS01 and CqSRS02 may also be regulated by auxin signaling (Singh et al. 2020). Studies in rice showed that OsSHI1 inhibited the transcriptional activity of IPA1, regulated plant structure, improved stem strength and increased the number of branches by affecting the DNA binding activity of IPA1 on the promoter region of OSTB1 and OSDEP1 (Duan et al. 2019). In Arabidopsis thaliana, SHI gene plays a role in the regulation of stamen development, cell amplification and flowering time (Veronika et al. 2012). So the five CqSRS genes that are highly similar may have similar functions.

Gene expression pattern is closely related to gene function. In this study, the expression levels of most genes were significantly increased under different treatments (SA, low temperature and $\mathrm{NaCl}$ ). Previous studies showed that SRS gene (LOC_Os01g72490) in maize could be induced by GA, but inhibited by PB, indicating that GA and PB activate antagonistic mechanism (Yang et al. 2020). It shows that SRS genes play an important role in plant development regulation and response to abiotic stress; they may be involved in the regulation of various responses related to stress and hormones. It was also found that the expression patterns of SRS genes in quinoa were different, indicating that these genes may participate in different biological processes or play different biological functions. Most genes are a low expression in the leaves; this is consistent with studies in Arabidopsis (Kim et al. 2010). Although some genes were homologous, their expression levels in roots and leaves were quite different, indicating that some SRS genes showed tissue dependence (CqSRS01 and CqSRS08). Meanwhile, some subfamilies have different gene expression patterns; it is speculated that the difference in the expression of different SRS between the same subfamily may be related to the sequence out of the conservative motif. Studies have shown that OsSHI1 in rice is highly expressed in roots but not in leaves, and some AtSRS genes are highly expressed in flowers and roots but not in leaves (Duan et al. 2019; Kuusk et al. 2006). These results are consistent with this study. 


\section{Conclusion}

Finally, a total of 10 SRS genes were identified in quinoa. Phylogenetic tree analysis shows that CqSRS genes are divided into three evolutionary groups, and the gene structure shows that the number of exons of CqSRS is between 2-5. The gene expansion of this family may be the result of fragment duplication. Promoter analysis revealed that there are a total of 44 elements related to plant hormone response, light response, stress response, and tissue-specific expression. Transcriptome data analysis shows that CqSRS genes have different expression patterns, qRT-PCR shows that all SRS family genes are responsive to SA, $\mathrm{NaCl}$ and low temperature. These results indicate that the main expression patterns and detailed functions of quinoa SRS genes are different in different developmental stages. Therefore, future research on these CqSRS genes may reveal the different functions of quinoa SRS genes. This study can further deepen our understanding of the molecular evolution and function of the quinoa SRS gene family, and provide a theoretical basis for further research on the SRS family in plants.

\section{Declarations}

Acknowledgement: We would like to thank the reviewers and editors for careful reading and helpful comments on this manuscript.

Funding Statement: This project was funded by the National Natural Science Foundation of China (32060401); Fostering Foundation for the Excellent Ph.D. Dissertation of Gansu Agricultural University [YB2021001].

\section{Compliance with ethical standards}

Conflict of Interest The authors stated that they had no interest which might be perceived as posing a conflict or bias.

Ethical approval This article does not contain any studies with human participants or animals performed by any of the authors.

\section{Author Contributions}

Conceptualization: Xiao-Lin Zhou, Xiao-Hong Wei

Formal analysis: Xiao-Lin Zhu.

Funding acquisition: Xiao-Hong Wei.

Investigation: Xiao-Lin Zhu, Bao-Qiang Wang, Xian Wang.

Methodology: Xiao-Lin Zhu, Xian Wang.

Software: Xiao-Lin Zhu, Bao-Qiang Wang, Xian Wang. 
Visualization: Xiao-Lin Zhu, Xian Wang

Writing - original draft: Xiao-Lin Zhu.

Writing - review \& editing: Xiao-Hong Wei.

\section{References}

1. Airoldi CA, Davies B(2012)Gene Duplication and the Evolution of Plant MADS-box Transcription Factors. Journal of Genetics and Genomics 39, 157-165

2. Bert DR, Dominique A, Wei X, Paul O, Lucia CS, Stefan K (2012) A role for the root cap in root branching revealed by the non-auxin probe naxillin. Nat Chem Biol 8:798-805

3. Boyer JS (1982) Plant productivity and environment. Science 218:443-448

4. Cao SQ, Guo MJ, Wang C, Xu WJ, Shi TY, Tong G (2019) Genome-wide characterization of aspartic protease (AP) gene family in Populus trichocarpa and identification of the potential PtAPs involved in wood formation. BMC Plant Biol 19:276

5. Chen CJ, Chen H, Zhang Y, Thomas HR, Frank MH, He YH (2020) TBtools, a Toolkit for Biologists integrating various HTS-data handling tools with a user-friendly interface. Mol Plant 13:1194-1202

6. Combet C, Blanchet C, Geourjon C, Deléage G (2000) NPS@: network protein sequence analysis. Trends Biochem Sci 25:147-150

7. David EJ, Yung SH, Damien JL, Sandra MS, Bo L, Theo JAB (2017) Corrigendum: The genome of Chenopodium quinoa. Nature 545:510

8. Duan E, Wang Y, Li X, Lin Q, Zhang T, Wang Y (2019) OsSHI1 regulates plant architecture through modulating the transcriptional activity of IPA1 in rice. Plant Cell 31:1026-1042

9. Fan K, Wang M, Miao Y, Ni M, Bibi N, Yuan S (2014) Molecular evolution and expansion analysis of the NAC transcription factor in Zea mays. PLoS ONE 9:e111837

10. Finn RD, Bateman A, Clements J, Coggill P, Eberhardt RY, Eddy SR (2014) Pfam: the protein families database. Nucleic Acids Res 42:D222-D230

11. Fridborg I, Kuusk S, Moritz T, Sundberg E (1999) The Arabidopsis dwarf mutant shi exhibits reduced gibberellin responses conferred by overexpression of a new putative zinc finger protein. Plant Cell 11:1019-1031

12. Fridborg I, Kuusk S, Robertson M, Sundberg E (2001) The Arabidopsis protein SHI represses gibberellin responses in Arabidopsis and barley. Plant Physiol 127:937-948

13. Gardy JL, Laird MR, Chen F, Rey S, Walsh CJ, Ester M (2005) PSORTb v.2.0: expanded prediction of bacterial protein subcellular localization and insights gained from comparative proteome analysis. Bioinformatics 21:617-623

14. Gasteiger E, Hoogland C, Gattiker A, Duvaud SE, Wilkins MR, Appel RD (1999) Protein identification and analysis tools on the ExPASy server. Methods Mol Biol 112:531-552 
15. Gomariz-Fernández A, Sánchez-Gerschon V, Fourquin C, Ferrándiz C (2017) The Role of SHI/STY/SRS Genes in Organ Growth and Carpel Development Is Conserved in the Distant Eudicot Species Arabidopsis thaliana and Nicotiana benthamiana. Front Plant Science 8:814

16. Greb T, Clarenz O, Schafer E, Muller D, Herrero R, Schmitz G (2003) Molecular analysis of the LATERAL SUPPRESSOR gene in Arabidopsis reveals a conserved control mechanism for axillary meristem formation. genes development 17:1175-1187

17. He B, Shi PB, Lv YD, Gao ZP, Chen GX (2020) SRS Gene coexpression network analysis reveals the role of genes in senescence leaf of maize. Journal of Genetics and Genomics 99, undefined

18. Hossain MS, Shrestha A, Zhong SH, Miri M, Austin RS, Sato S (2016) Lotus japonicus NF-YA1 Plays an Essential Role During Nodule Differentiation and Targets Members of the SHI/STY Gene Family. Mol Plant Microbe Interact 29:950-964

19. Hu B, Jin J, Guo AY, Zhang H, Luo J, Gao G (2015) GSDS 2.0: an upgraded gene feature visualization server. Bioinformatics 31:1296-1297

20. Ive DS, Valya V, Bert DR, Mitchell PL, Wim G, Daniël VD (2008) Receptor-like kinase ACR16 restricts formative cell divisions in the Arabidopsis root. Science 322:594-597

21. Jarvis DE, Ho YS, Lightfoot DJ, Schmöckel SM, Li B, Borm TJA (2017) The genome of Chenopodium quinoa. Nature 542:307-312

22. Kelley LA, Mezulis S, Yates CM, Wass MN, Sternberg MJE (2015) The Phyre2 web portal for protein modeling, prediction and analysis. Nat Protoc 10:845

23. Kim SG, Lee S, Kim YS, Yun DJ, Woo JC, Park CM (2010) Activation tagging of an Arabidopsis SHIRELATED SEQUENCE gene produces abnormal anther dehiscence and floral development. Plant Mol Biol 74:337-351

24. Klund DM, Staldal V, Valsecchi I, Cierlik I, Eriksson C, Hiratsu K (2010) The Arabidopsis thaliana STYLISH1 protein acts as a transcriptional activator regulating auxin biosynthesis. Plant Cell 22:349363

25. Kumar S, Stecher G, Tamura K (2016) MEGA7: molecular evolutionary genetics analysis version 7.0 for bigger datasets. Mol Biol Evol 33:1870-1874

26. Kuusk S, Sohlberg JJ, Magnus E, klund D, Sundberg E (2006) Functionally redundant SHI family genes regulate Arabidopsis gynoecium development in a dose-dependent manner. Plant journal 47:99-111

27. Larkin MA, Blackshields G, Brown NP, Chenna R, McGettigan PA, McWilliam H (2007) Clustal W and Clustal X version 2.0. Bioinformatics 23:2947-2948

28. Lescot M, Dehais P, Thijs G, Marchal K, Moreau Y, Van PY (2002) PlantCARE, a database of plant cisacting regulatory elements and a portal to tools for in silico analysis of promoter sequences. Nucleic Acids Res 30:325-327

29. Librado P, Rozas J (2009) DnaSP v5: a software for comprehensive analysis of DNA polymorphism data. Bioinformatics 25:1451-1452

30. Liu X, Chu Z (2015) Genome-wide evolutionary characterization and analysis of bZIP transcription factors and their expression profiles in response to multiple abiotic stresses in Brachypodium 
distachyon. BMC Genom 16:227

31. Liu Y, Jiang HY, Chen W, Qian Y, Ma Q, Cheng B (2011) Genome-wide analysis of the auxin response factor (ARF) gene family in maize (Zea mays). Plant Growth Regul 63:225-234

32. Livak KJ, Schmittgen TD (2001) Analysis of Relative Gene Expression Data using Real-Time Quantitative PCR. Method 25:402-408

33. Lynch M, Conery JS (2003) The evolutionary demography of duplicate genes. J Struct Funct Genomics $3: 35-44$

34. Mao H, Yu L, Li Z, Liu H, Han R (2016) Molecular evolution and gene expression differences within the HD-Zip transcription factor family of Zea mays L. Genetica 144:243-257

35. Marchler-Bauer A, Lu S, Anderson JB, Chitsaz F, Derbyshire MK, DeWeese-Scott C (2011) CDD: a conserved domain database for the functional annotation of proteins. Nucleic Acids Res 39:225-229

36. McGregor N, Yin V, Tung CC, Van Petegem F, Brumer H (2017) Crystallographic insight into the evolutionary origins of xyloglucan endotransglycosylases and endohydrolases. Plant J 89:651-670

37. Poole RL (2017) The TAIR database. Methods Molecular in Biology 406:179-212

38. Riechmann JL, Heard J, Martin G, Reuber L, Jiang C, Keddie J (2000) Arabidopsis transcription factors:genome wide comparative analysis among eukaryotes. Science 290:2105-2110

39. Rogozin IB, Lyons-Weiler J, Koonin EV (2000) Intron sliding in conserved genefamilies. Trends Genet $16: 430-432$

40. Schultz J, Copley RR, Doerks T, Ponting CP, Bork P (2000) SMART: a web-based tool for the study of genetically mobile domains. Nucleic Acids Res 28:231-234

41. Singh S, Yadav S, Singh A, Mahima M, Singh A, Gautam V (2020) Auxin signaling modulates LATERAL ROOT PRIMORDIUM1 (LRP1) expression during lateral root development in Arabidopsis. Plant J 101:87-100

42. Sohlberg JJ, Myrenas M, Kuusk S, Lagercrantz U, Kowalczyk M, Sandberg G (2006) STY1 regulates auxin homeostasis and affects apical-basal patterning of the Arabidopsis gynoecium. Plant journal 47:112-123

43. Steven DC (1996) Molecular genetic studies confirm the role of brassinosteroids in plant growth and development. Plant J 10:1-8

44. Suyama M, Torrents D, Bork P (2006) PAL2NAL: robust conversion of protein sequence alignments into the corresponding codon alignments. Nucleic Acids Res 34:W609-W612

45. Szklarczyk D, Franceschini A, Wyder S, Forslund K, Heller D, Huerta-Cepas J (2015) STRING v10: Proteinprotein interaction networks, integrated over the tree of life. Nucleic Acids Res 43:D447-D452

46. Tian F, Yang DC, Meng YQ, Jin JP, Gao G (2019) PlantRegMap: charting functional regulatory maps in plants. Nucleic Acids Research, gkz1020

47. Veronika S, Izabela C, Song C, Katarina L, Tammy B, Mattias M (2012) The Arabidopsis thaliana transcriptional activator STYLISH1 regulates genes affecting stamen development, cell expansion and timing of flowering. Plant Mol Biol 78:545-559 
48. Wang YS, Zhang JL, Hu ZL, Guo XH, Tian SB, Chen GP (2019) Genome-Wide Analysis of the MADS-Box Transcription Factor Family in Solanum lycopersicum. Int J Mol Sci 20:2961

49. Yang J, Xu P, Yu D (2020) Genome-Wide Identification and Characterization of the SHI-Related Sequence Gene Family in Rice. Evolutionary Bioinformatics 16:1176934320941495

50. Yuan TT, Xu HH, Zhang Q, Zhang LY, Lu YT (2018) The COP1 Target SHI-RELATED SEQUENCE5 Directly Activates Photomorphogenesis-Promoting Genes. Plant Cell 30:2368-2382

51. Zhang H, Gao S, Lercher MJ, Hu S, Chen WH (2012) EvolView, an online tool for visualizing, annotating and managing phylogenetic trees. Nucleic Acids Research, W569-72

52. Zhang S, Xu R, Luo X, Jiang Z, Shu H (2013) Genome-wide identification and expression analysis of MAPK and MAPKK gene family in Malus domestica. Gene 531:377-387

53. Zhang X, Cheng T, Wang G, Yan Y (2013) Cloning and evolutionary analysis of tobacco MAPK gene family. Mol Biol Rep 40:1407-1415

54. Zhang Y, Behrens I, Zimmermann R, Ludwig Y, Hey S, Hochholdinger F (2015) LATERAL ROOT PRIMORDIA 1 of maize acts as a transcriptional activator in auxin signalling downstream of the Aux/IAA gene rootless with undetectable meristem 1. Journal of Experimental botany 66:3855-3863

55. Zhao W, Liu YW, Zhou JM, Zhao SP, Zhang XH, Min DH (2016) Genome-wide analysis of the lectin receptor-like kinase family in foxtail millet (Setaria italica L.). Plant Cell Tissue Organ Culture 127:335346

56. Zou CS, Chen AJ, Xiao LH, Muller HK, Peter A, Georg H (2017) A high-quality genome assembly of quinoa provides insights into the molecular basis of salt bladder-based salinity tolerance and the exceptional nutritional value. Cell Res 27:1327-1340

\section{Tables}

Table 1. Characteristics of SRS genes in quinoa 


\begin{tabular}{|llllllll|}
\hline $\begin{array}{l}\text { Gene } \\
\text { accession No }\end{array}$ & Gene & $\begin{array}{l}\text { Size } \\
\text { (aa) }\end{array}$ & $\begin{array}{l}\text { Molecular } \\
\text { weight } \\
\text { (D) }\end{array}$ & $\begin{array}{l}\text { Isoelectric } \\
\text { point }\end{array}$ & $\begin{array}{l}\text { Instability } \\
\text { index }\end{array}$ & GRAVY & $\begin{array}{l}\text { Subcellular } \\
\text { Localization }\end{array}$ \\
\hline $\begin{array}{l}\text { AUR62000185- } \\
\text { RA }\end{array}$ & CqSRS01 & 370 & 37758.52 & 8.33 & 36.33 & -0.452 & $\begin{array}{l}\text { plasma } \\
\text { membrane }\end{array}$ \\
\hline $\begin{array}{l}\text { AUR62006536- } \\
\text { RA }\end{array}$ & CqSRS02 & 241 & 24289.62 & 8.90 & 39.45 & -0.487 & nucleus \\
$\begin{array}{l}\text { AUR62007206- } \\
\text { RA }\end{array}$ & CqSRS03 & 312 & 35034.26 & 6.80 & 52.91 & -0.854 & mitochondrion \\
\hline $\begin{array}{l}\text { AUR62007636- } \\
\text { RA }\end{array}$ & CqSRS04 & 244 & 26367.17 & 5.56 & 45.09 & -0.491 & cytoplasm \\
\hline $\begin{array}{l}\text { AUR62007664- } \\
\text { RA }\end{array}$ & CqSRS05 & 246 & 26717.52 & 5.45 & 47.68 & -0.591 & cytoplasm \\
\hline $\begin{array}{l}\text { AUR62010428- } \\
\text { RA }\end{array}$ & CqSRS06 & 143 & 15336.89 & 5.92 & 52.51 & -0.290 & cytoplasm \\
\hline $\begin{array}{l}\text { AUR62014445- } \\
\text { RA }\end{array}$ & CqSRS07 & 170 & 17896.76 & 6.08 & 40.35 & -0.449 & mitochondrion \\
\hline $\begin{array}{l}\text { AUR62016794- } \\
\text { RA }\end{array}$ & CqSRS08 & 246 & 26764.40 & 4.81 & 32.45 & -0.539 & cytoplasm \\
\hline $\begin{array}{l}\text { AUR62018795- } \\
\text { RA }\end{array}$ & CqSRS09 & 312 & 34969.13 & 5.85 & 51.62 & -0.799 & mitochondrion \\
\hline $\begin{array}{l}\text { AUR62034552- } \\
\text { RA }\end{array}$ & CqSRS10 & 163 & 16857.59 & 5.60 & 37.67 & -0.374 & cytoplasm \\
\hline
\end{tabular}

Note: GRAVY represents Grand average of hydropathicity.

Table 2. Gene duplication in CqSRS family in quinoa

\begin{tabular}{|c|c|c|c|c|c|c|c|}
\hline $\begin{array}{l}\text { Duplicated } \\
\text { CAMTA gene1 }\end{array}$ & $\begin{array}{l}\text { Duplicated } \\
\text { CAMTA gene2 }\end{array}$ & $\mathrm{Ka}$ & Ks & $\mathrm{Ka} / \mathrm{Ks}$ & $\begin{array}{l}\text { Date(MYA)T= } \\
\mathrm{Ks} / 2 \lambda\end{array}$ & $\begin{array}{l}\text { Selective } \\
\text { pressure }\end{array}$ & $\begin{array}{l}\text { Duplicate } \\
\text { type }\end{array}$ \\
\hline CqSRS03 & CqSRS09 & 0.021 & 0.116 & 0.180 & 6.830 & $\begin{array}{l}\text { Purifying } \\
\text { selection }\end{array}$ & Segmental \\
\hline CqSRSO4 & CqSRS05 & 0.049 & 0.185 & 0.263 & 10.959 & $\begin{array}{l}\text { Purifying } \\
\text { selection }\end{array}$ & Segmental \\
\hline CqSRS06 & CqSRS08 & 0.174 & 0.239 & 0.728 & 14.151 & $\begin{array}{l}\text { Purifying } \\
\text { selection }\end{array}$ & Segmental \\
\hline CqSRS07 & CqSRS10 & 0.011 & 0.116 & 0.098 & 6.836 & $\begin{array}{l}\text { Purifying } \\
\text { selection }\end{array}$ & Segmental \\
\hline
\end{tabular}

Note: The non-synonymous (Ka) and synonymous substitution rate (Ks); millions of years ago (MYA) 


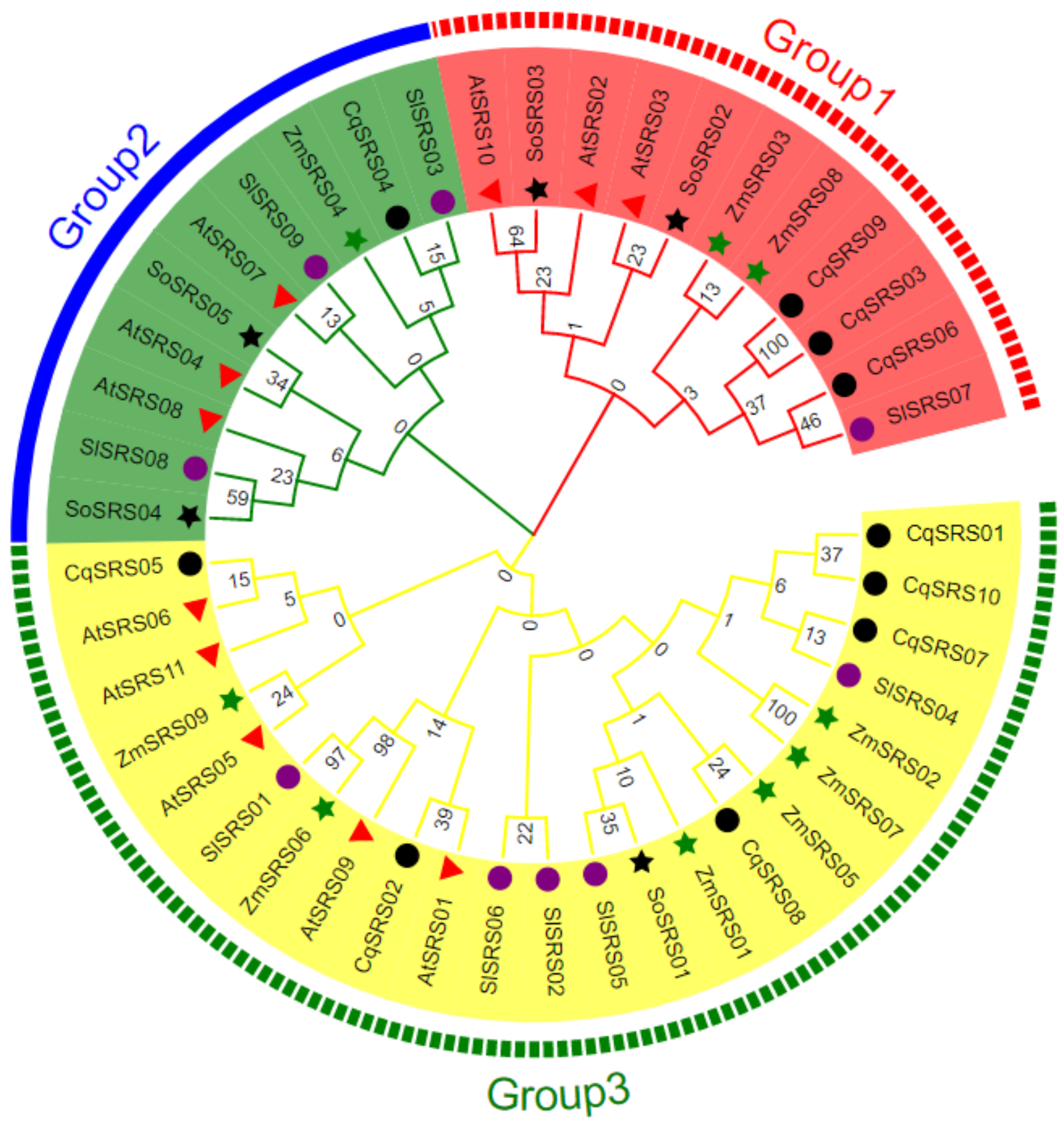

Figure 1

Phylogenetic relationships of SRS proteins from Arabidopsis $₫$ Zea mays L $\square$ Solanum lycopersicum $\square$ Spinacia oleracea $L$ and quinoa. The proteins clustered into four subgroups, denoted with different colors to represent subfamilies as follows: Group1 (red), Group 2 (blue), Group 3 (yellow). The information of the SRS family members from Arabidopsis $\square$ Solanum lycopersicum $\square$ Zea mays $L$ and Spinacia oleracea $L$ is listed in the supporting information (Supplementary Table S1). Among them, the red triangle represents Arabidopsis, the black circle represents quinoa, the green star represents Zea mays $L$, the purple circle represents Solanum lycopersicum, the black star represents Spinacia oleracea L. The numbers in the clades are posterior probability values. Bootstrap values based on 1,000 replications were calculated. 


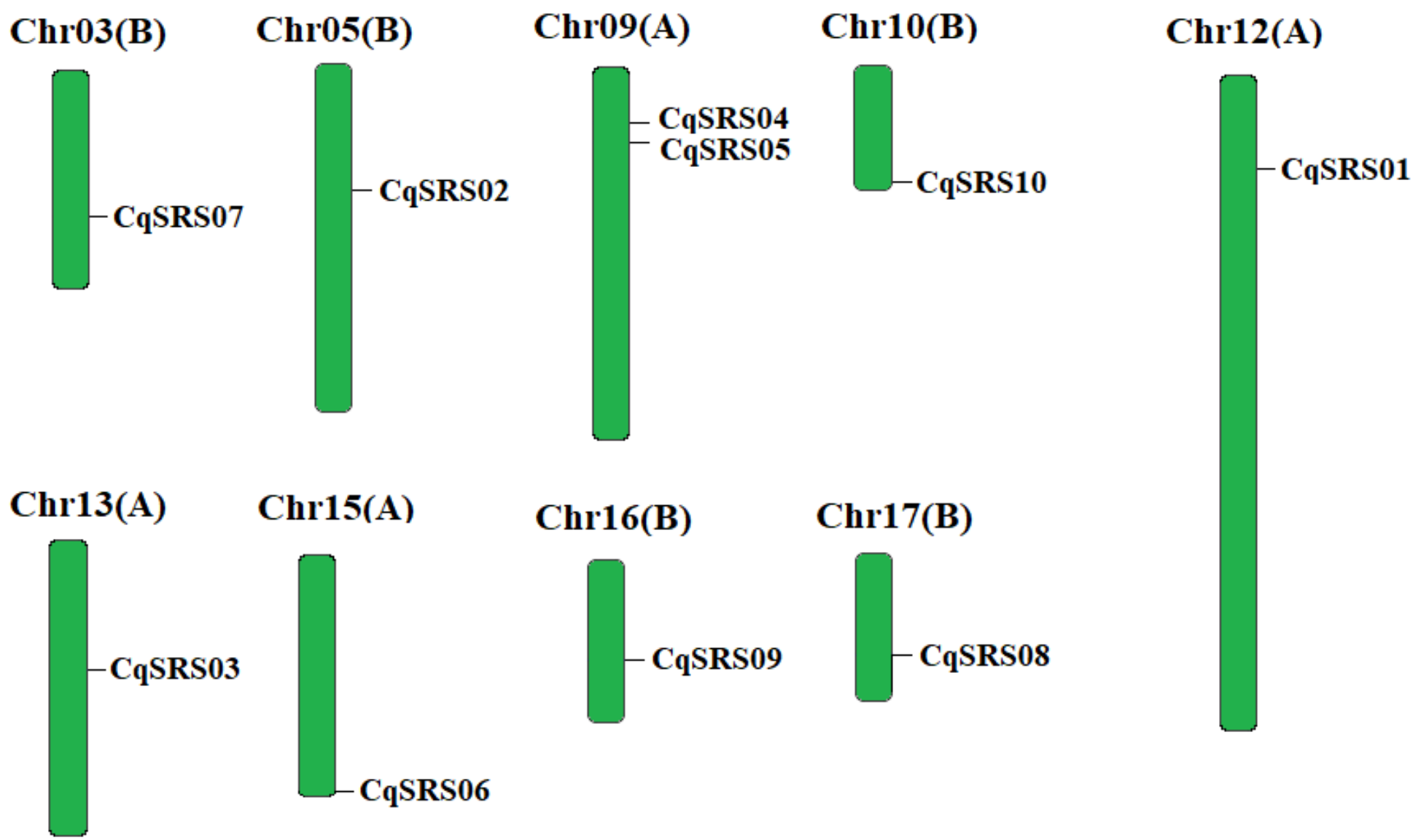

Figure 2

Chromosome mapping of CqSRS genes in quinoa. 
A

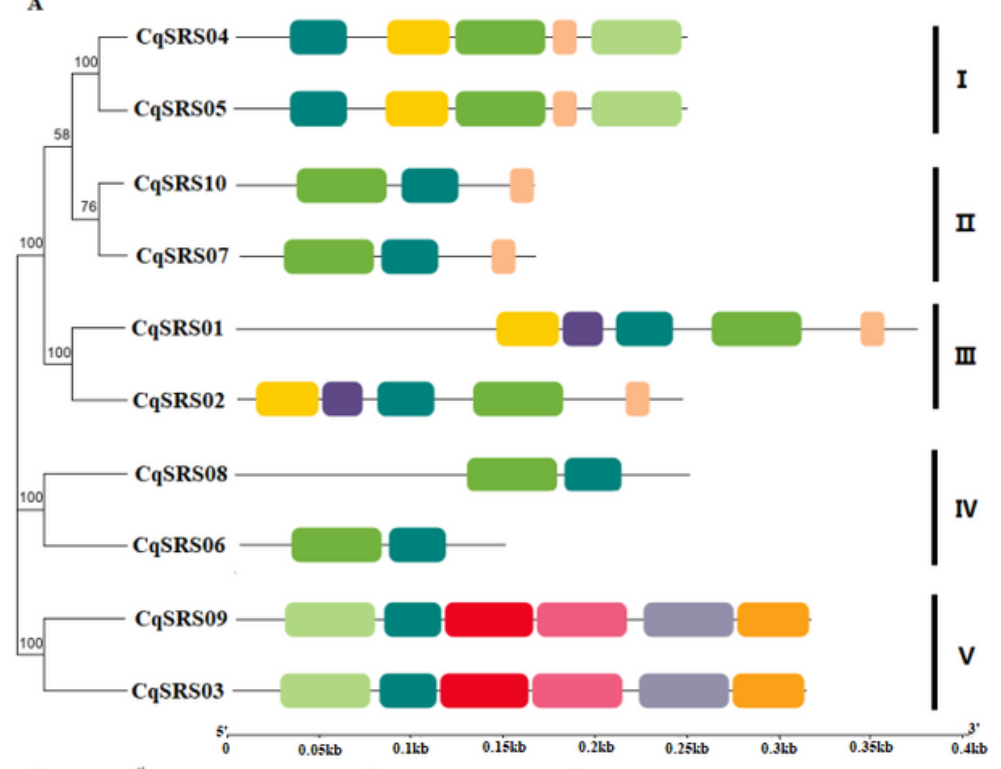

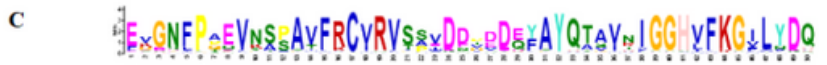
Motif1

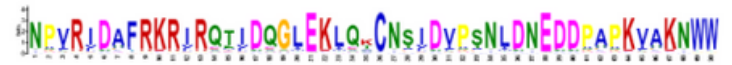
Motif3

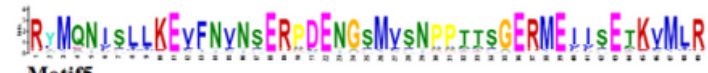
Motifs

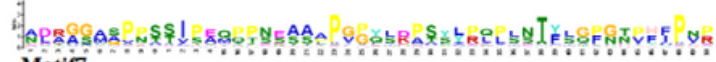
Motif

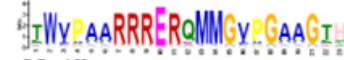

Motify
B
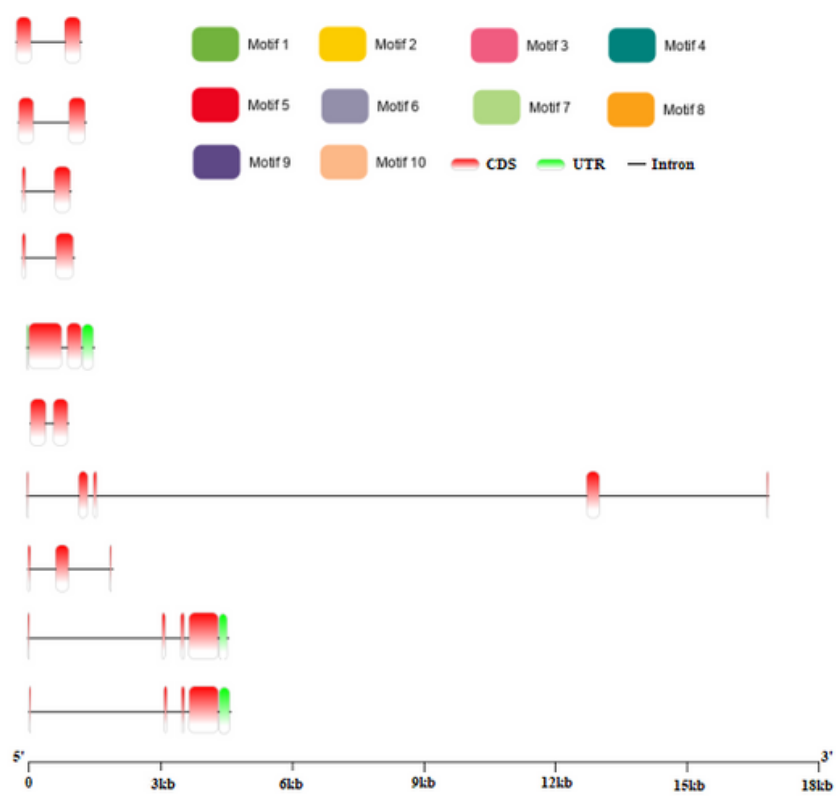

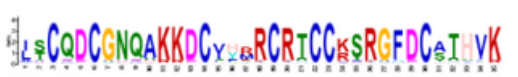

Motif2

月.

Motif4

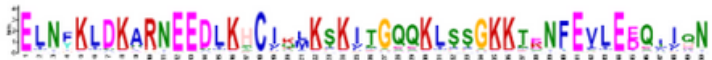

Motif6

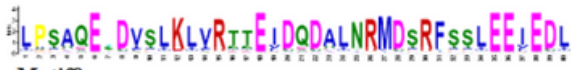

Motif8

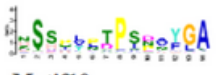

Motif10

Figure 3

Structural analysis of CqSRS genes in quinoa. An unrooted phylogenetic tree was constructed based on the full-length sequences of CqSRS proteins using the N-J method in MEGA7. Bootstrap values based on 1,000 replications were calculated. (A) The distribution of motif in SRS proteins. (B) The exon-intron structure of the SRS gene. (C) The amino acid composition of each motif, motif sequences in S2 Table. 


\begin{tabular}{|c|c|c|c|c|c|c|c|c|c|c|c|}
\hline ABRE & 7 & 3 & 5 & 4 & 3 & 1 & 7 & 1 & 1 & 8 & \multirow{11}{*}{ Phytohormone responsiveness } \\
\hline AuxRE & & & & 1 & 1 & & & 1 & & & \\
\hline AuxRR-core & & & & 1 & & & & & & & \\
\hline CGTCA-motif & 2 & 2 & 2 & 3 & 2 & 2 & 1 & 1 & 6 & 1 & \\
\hline GARE-motif & & & & & 1 & & 1 & 1 & & 2 & \\
\hline P-box & & 2 & & & & 1 & 1 & & & 1 & \\
\hline TATC-box & & & & & & 2 & & & & 1 & \\
\hline TCA-element & 1 & 3 & & 5 & 3 & & 1 & 1 & & 1 & \\
\hline TGA-box & & & & & & & & & 1 & & \\
\hline TGACG-motif & 2 & 2 & 2 & 3 & 2 & 2 & 1 & 1 & 6 & 1 & \\
\hline TGA-element & & 1 & 2 & & & 1 & & & 1 & & \\
\hline ARE & 4 & 6 & 5 & 1 & 3 & 1 & 4 & 3 & 3 & 3 & \multirow{8}{*}{ Stress responsiveness } \\
\hline O2-site & & & 1 & & & 2 & & 1 & 2 & 1 & \\
\hline CAT-box & & & & & & & & 1 & & 1 & \\
\hline GCN4_motif & & & & 1 & & & & & & & \\
\hline AACA_motif & 1 & 1 & & & & & & & & & \\
\hline GC-motif & 1 & 1 & 1 & & & & 1 & & & 1 & \\
\hline MBSI & 1 & & & & & & & & & & \\
\hline MSA-like & & & & & & & 1 & & & 1 & \\
\hline TC-rich repeats & 1 & & 3 & 2 & 1 & & 1 & 1 & 2 & 2 & \multirow{3}{*}{ Tissue-specific expression } \\
\hline LTR & 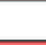 & & & & & & 2 & 1 & 3 & 2 & \\
\hline MBS & 1 & & 1. & & & & 1 & & 1. & 1 & \\
\hline 3-AF1 binding site & & & & & & & & 1 & 1 & 1 & \multirow{22}{*}{ Light responsiveness } \\
\hline AAAC-motif & 1 & 1 & & & & & & & & & \\
\hline ACE & & & 1 & & 1 & 1 & & & 1 & & \\
\hline AE-box & & 1 & & & 1 & & & 1 & & & \\
\hline AT1-motif & & & & & 1 & & & 2 & & & \\
\hline ATC-motif & 1 & & & 1 & & & & & & & \\
\hline ATCT-motif & & & 1 & 1 & 1 & & & 1 & & 1 & \\
\hline Box 4 & 4 & 3 & 1 & 4 & 3 & 2 & 3 & 1 & 10 & 3 & \\
\hline Box II & & & & & & & 1 & & & & \\
\hline chs-CMA1a & 1 & & & & 1 & & & & & & \\
\hline chs-CMA2a & & & & & & & & 1 & & & \\
\hline GA-motif & 1 & 1 & & & & & & & 1 & & \\
\hline GATA-motif & 1 & & 1 & & & 3 & 1 & 1 & 2 & 1 & \\
\hline GATT-motif & & & & 1 & 1 & & & & & & \\
\hline G-Box & 7 & 3 & 9 & 4 & 3 & 2 & 10 & 1 & 1 & 8 & \\
\hline GT1-motif & & & 2 & 6 & 3 & 3 & 4 & 4 & 3 & 4 & \\
\hline I-box & & 2 & 3 & 3 & & 4 & & 1 & & & \\
\hline L-box & & & & & & 1 & & & & & \\
\hline MRE & 2 & 1 & & & & & 1 & 1 & & 1 & \\
\hline Sp1 & & & & & & & & 1 & & & \\
\hline TCCC-motif & & & 1 & & & & & & 1 & & \\
\hline TCT-motif & 2 & 2 & 1 & 3 & 4 & 3 & 3 & 1 & 1 & 5 & \\
\hline
\end{tabular}

Figure 4

Cis-acting components of quinoa SRS genes. All promoter sequences (2000bp) were analyzed. Cis-acting element names and functions can be found in S3 


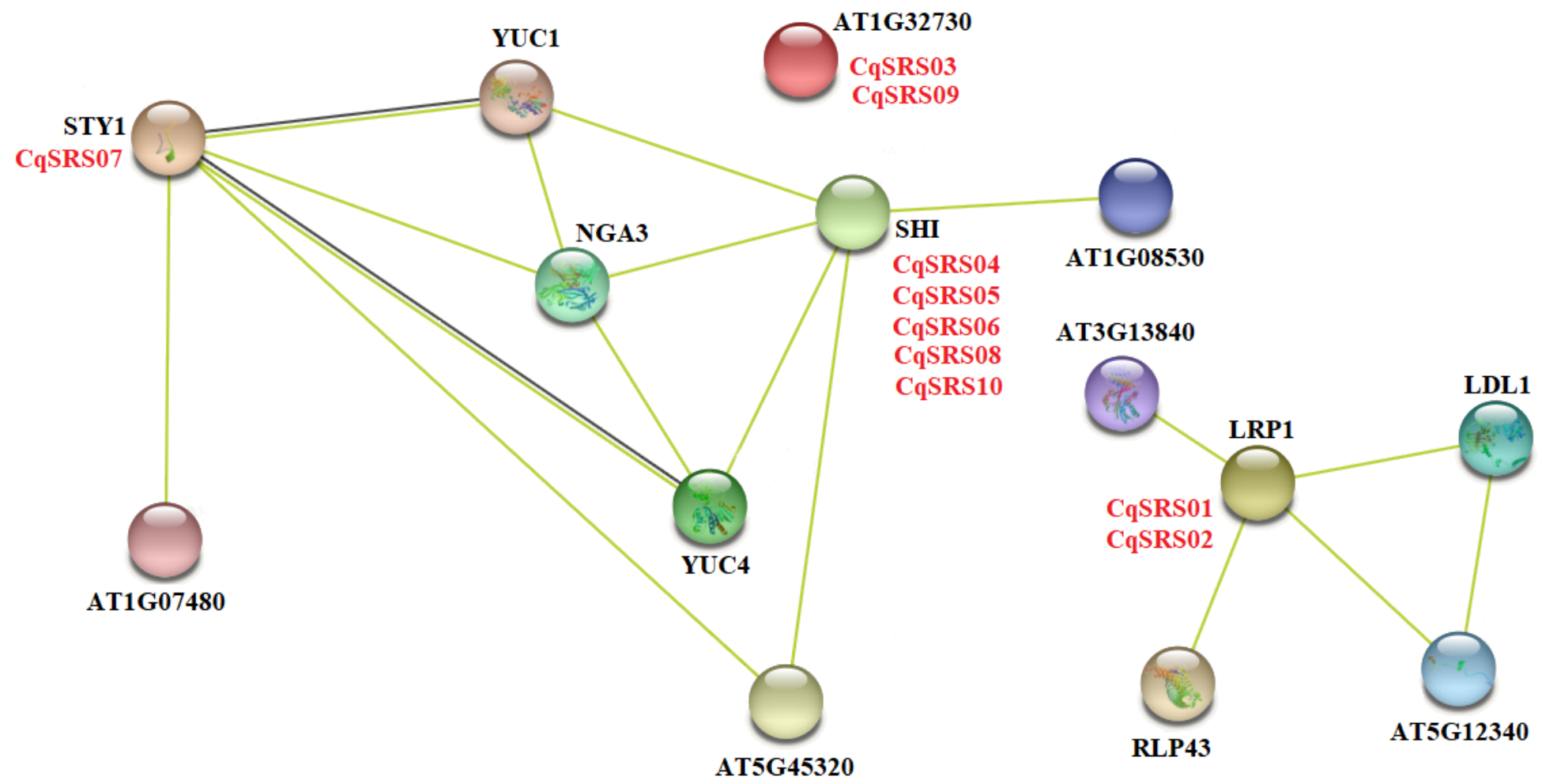

\section{Figure 5}

The potential interaction network of CqSRS based on the Arabidopsis and quinoa.

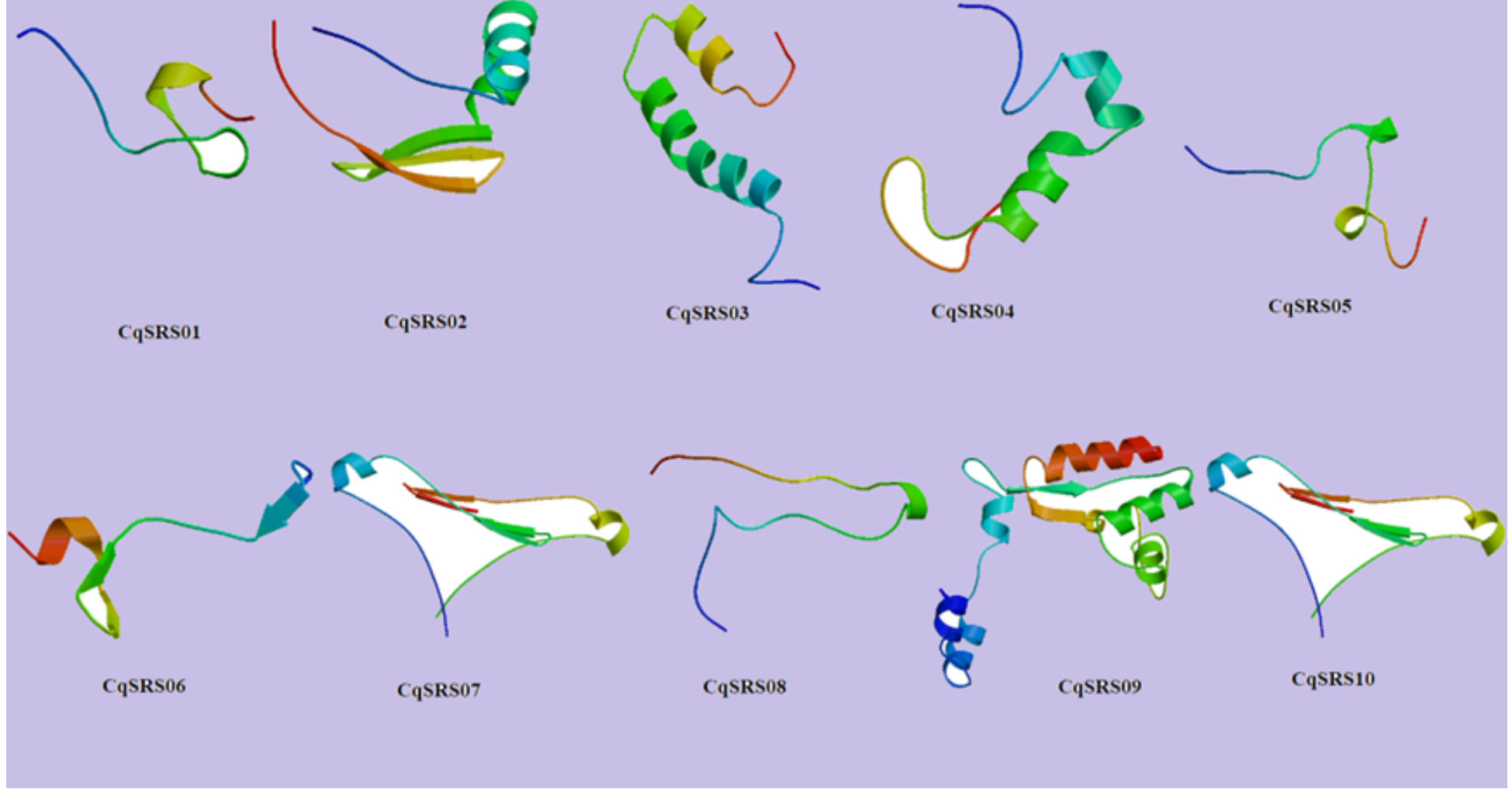

\section{Figure 6}

Tertiary Structure Prediction of SRS genes in quinoa. 
A

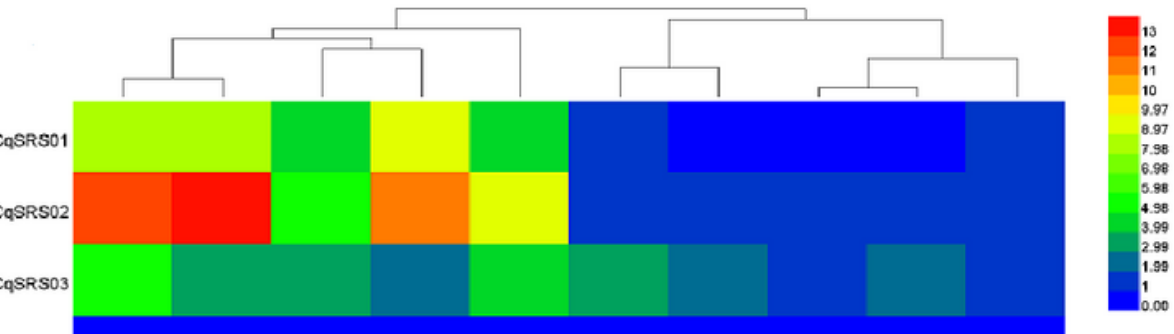

B

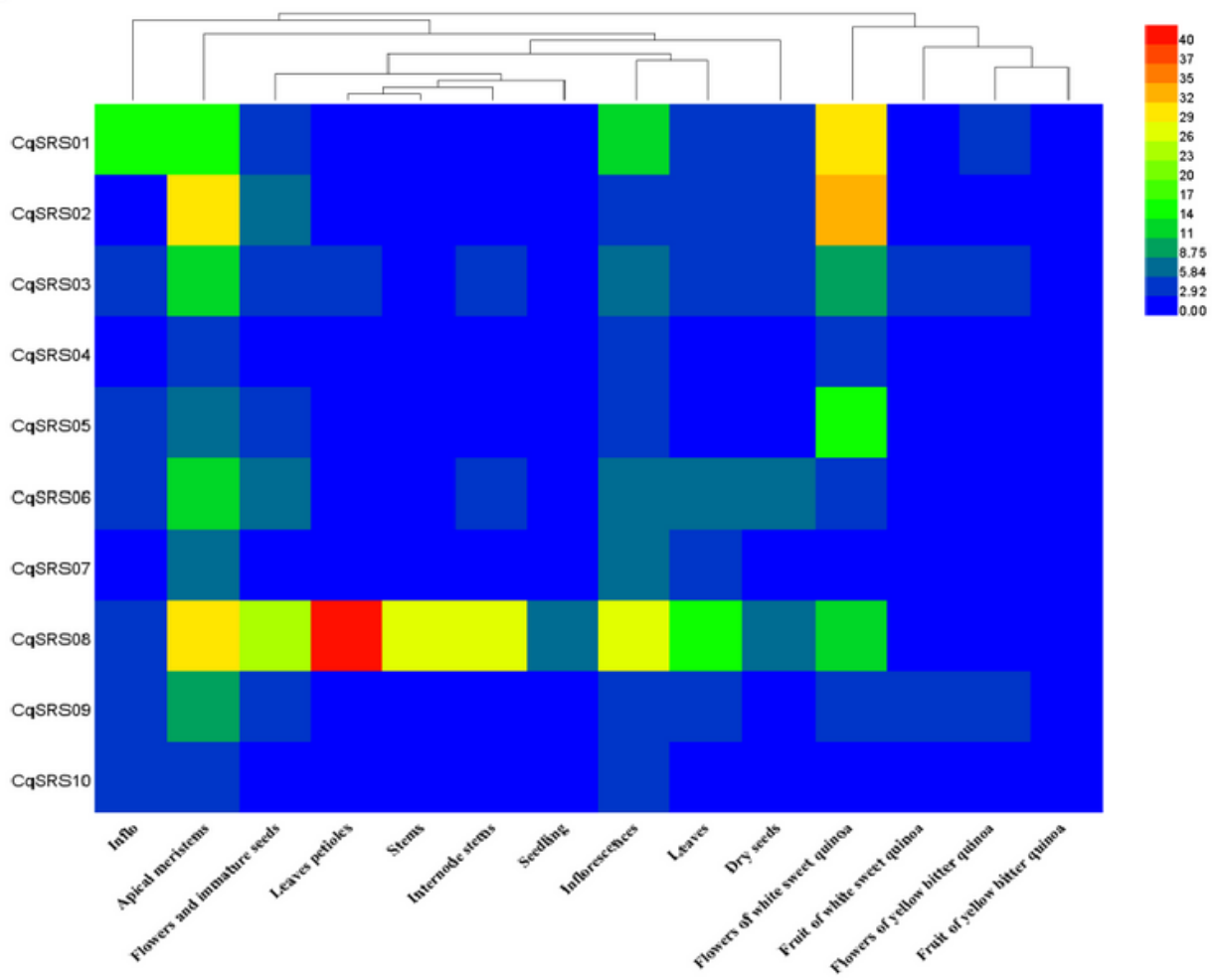

Figure 7

The expression profiles of SRS genes in different treatments $\rrbracket$ developmental stages and tissues of quinoa. (A) CqSRS expression patterns at different treatments. (B) CqSRS expression patterns at different developmental stages and tissues. Gene expression was calculated by FPKM. We standardized the data using the Log2 method. 

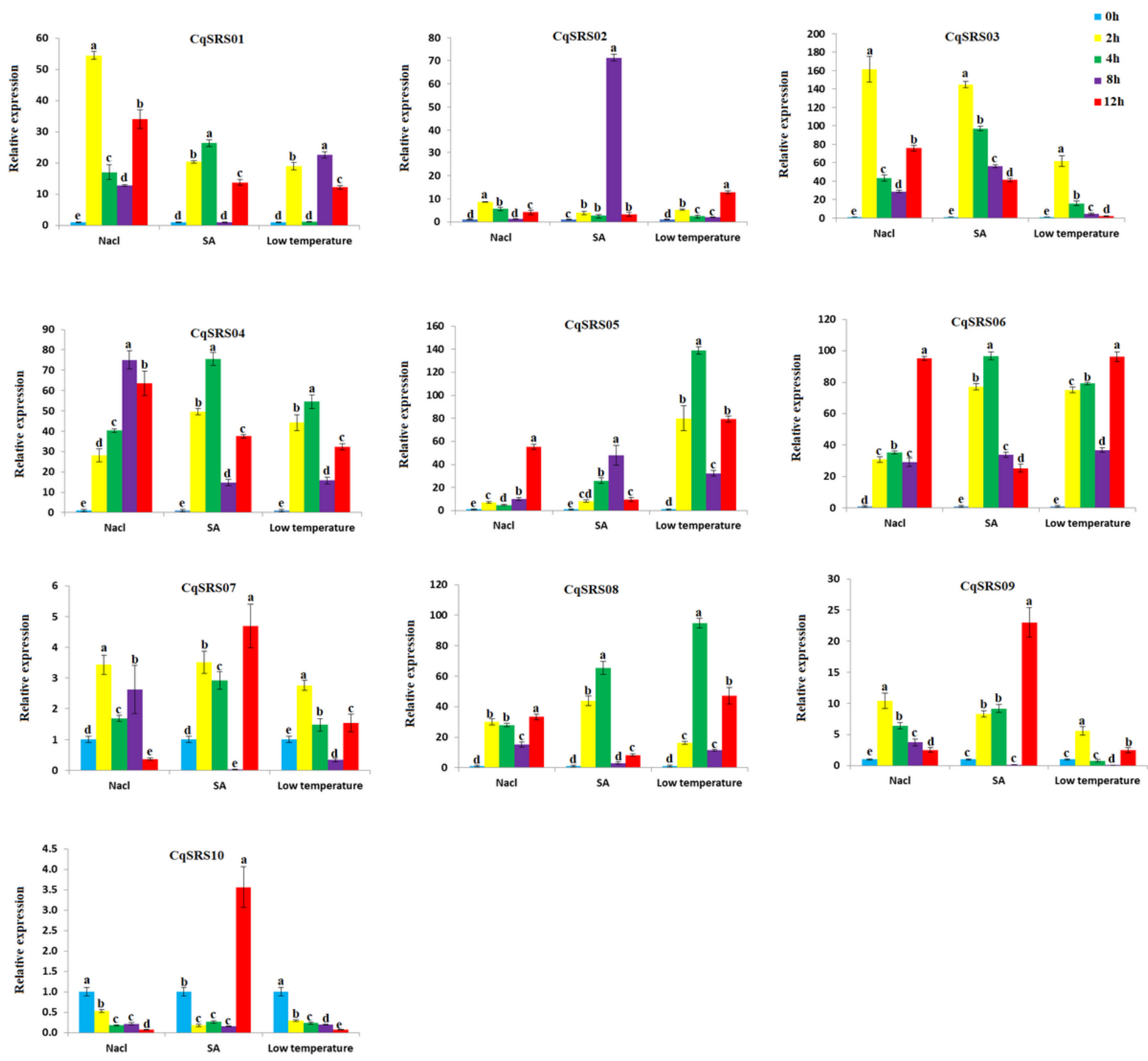

\section{Figure 8}

Expression profiles of 10 SRS genes using qRT-PCR analysis in quinoa. Values represented the mean \pm standard error of the mean (SEM) of three biological replicates with three technical replicates at different treatments. Error bars indicated the SEM among the three experiments.

\section{Supplementary Files}

This is a list of supplementary files associated with this preprint. Click to download. 
- S2Table.xIsx

- S3Table.xlsx

- S4Table.xlsx

- S5Table.xIsx

- S6Table.xlsx

- S7Table.xIsx 